\title{
ON THE ASYMPTOTIC PERFORMANCE OF ONE-BIT CO-ARRAY-BASED MUSIC
}

\author{
Saeid Sedighi ${ }^{\dagger}$, Bhavani Shankar ${ }^{\dagger}$, Mojtaba Soltanalian ${ }^{\ddagger}$, and Björn Ottersten ${ }^{\dagger}$ \\ ${ }^{\dagger}$ Interdisciplinary Centre for Security, Reliability and Trust (SnT), University of Luxembourg \\ $\ddagger$ Dept. of Electrical and Computer Engineering, University of Illinois at Chicago (UIC) \\ E-mails: \{saeid.sedighi,bhavani.shankar,bjorn.ottersten\}@uni.lu, msol@uic.edu
}

\begin{abstract}
Co-array-based Direction of Arrival (DoA) estimation using Sparse Linear Arrays (SLAs) has recently gained considerable attention in array processing thanks to its capability of providing enhanced degrees of freedom for DoAs that can be resolved. Additionally, deployment of one-bit Analog-to-Digital Converters (ADCs) has become an important topic in array processing, as it offers both a low-cost and a low-complexity implementation. Although the problem of DoA estimation form one-bit SLA measurements has been studied in some prior works, its analytical performance has not yet been investigated and characterized. In this paper, to provide valuable insights into the performance of DoA estimation from one-bit SLA measurements, we derive an asymptotic closed-form expression for the performance of One-Bit Co-Array-Based MUSIC (OBCAB-MUSIC). Further, numerical simulations are provided to validate the asymptotic closed-form expression for the performance of OBCAB-MUSIC and to show an interesting use case of it in evaluating the resolution of OBCAB-MUSIC.
\end{abstract}

Index Terms - One-bit quantization, sparse linear arrays, direction of arrival (DoA) estimation, performance evaluation.

\section{INTRODUCTION}

The problem of Direction of Arrival (DoA) estimation is of central importance in the field of array processing, with many applications in radar, sonar, and wireless communications [1-3]. Estimating DoAs using Uniform Linear Arrays (ULAs) is well investigated in the literature; a number of algorithms such as Maximum Likelihood (ML) estimation, MUSIC, ESPRIT and subspace fitting have been presented and their performance thoroughly analyzed [4-6]. However, it is widely known that ULAs are not capable of identifying more sources than the number of physical elements in the array [2, 6]. To transcend this limitation, exploitation of Sparse Linear Arrays (SLAs) with particular geometries, such as Minimum Redundancy Arrays (MRAs) [7], co-prime arrays [8] and nested arrays [9] has been proposed. These architectures can dramatically boost the degrees of freedom of the array for uncorrelated source signals such that a number of sources, significantly larger number than the number of physical elements in the array, can be identified. In addition, the enhanced degrees of freedom provided by these SLAs can improve the resolution performance appreciably compared to ULAs [9]. These features have spurred further research on DoA estimation using SLAs in recent years. A detailed study on DoA estimation via

This work is partially supported by the Luxembourg National Research Fund (FNR) under the ACCORDION project (reference number 11228830) and the BRIDGES project AWARDS as well as ERC AGNOSTIC (Grant ID: 742648), NSF 1704401, 1809225, and a DPI seed award.
SLAs through an analysis of the Cramér-Rao Bound (CRB) has been conducted in [10]. Further, several approaches for DoA estimation via SLAs have been proposed in the literature. In general, existing estimators can be classified under two main groups: 1 . Sparsity-Based Methods (SBMs), 2. Augmented Covariance-Based Methods (ACBMs). SBMs estimate DoAs by imposing sparsity constraints on source profiles and exploiting the compressive sensing recovery techniques [11-13]. However, in ACBMs, DoAs are estimated by applying conventional subspace methods such as MUSIC, ESPRIT on an Augmented Sample Covariance Matrix (ASCM) developed from the original sample covariance matrix by exploiting the difference co-array structure $[9,14,15]$. In addition, the authors of this paper recently proposed a Weighted Least Squares (WLS) estimator capable of asymptotically achieving the corresponding CRB for DoA estimation via SLAs [16].

The aforementioned techniques for DoA estimation via SLAs rest on the assumption that the analog array measurements are digitally represented using a significantly large number of bits per sample such that the resulting quantization errors can be disregarded. However, the use of high resolution Analog-to-Digital Converters (ADCs) is impractical in many modern applications due to limitations on power consumption and production cost. As a viable solution in such cases, deployment of low-resolution ADCs with a high sampling rate has been proposed in the literature [17-21]. Particularly, use of one-bit ADCs has received the most attention since they allow for sampling at an extremely high rate with a low cost and low power consumption. Recently, the problem of DoA estimation from one-bit data has gained considerable interest in the literature [2227]. This problem has been investigated in [22-25] when a ULA is employed and it has been shown that one-bit quantization leads to a moderate performance loss compared to the case where infinite-bit quantized data is used. Further, the problem of DoA estimation from one-bit SLA data has been addressed in [26, 27]; it has been demonstrated that the performance degradation due to one-bit quantization can, to some extent, be compensated using SLAs.

In this paper, to provide a better insight into the performance of DoA estimation using one-bit SLA measurements, we analytically study the performance of One-bit Co-Array-Based MUSIC (OBCAB-MUSIC) proposed in [26]. Specifically, we first provide an asymptotic closed-form expression for the performance of OBCAB-MUSIC. Then, we use this analytical expression for determining the performance limits of OBCAB-MUSIC including the impact of SNR and number of snapshots. Numerical simulations are provided to validate the derived asymptotic closed-form expression for the performance of OBCAB-MUSIC as well as to show an interesting use case of predicting the minimum source separation required for resolving of source DoAs using OBCAB-MUSIC.

Organization: Section 2 describes the system model. Sec- 
tion 3 reviews the one-bit co-array-based MUSIC algorithm. The asymptotic closed-form expression for the performance of OBCABMUSIC is given in Section 4. The simulation results and related discussions are included in Section 5. Finally, Section 6 concludes the paper.

Notation: Vectors and matrices are referred to by lower- and upper-case bold-face, respectively. The superscripts $*, T, H$ denote the conjugate, transpose and Hermitian (conjugate transpose) operations, respectively. $[\mathbf{A}]_{i, j}$ and $[\mathbf{a}]_{i}$ indicate the $(i, j)^{\text {th }}$ and $i^{\text {th }}$ entry of $\mathbf{A}$ and $\mathbf{a}$, respectively. $\hat{\mathbf{A}}$ and $\mathbf{a}$ denote the estimate of $\mathbf{A}$ and $\mathbf{a}$, respectively. $\left(a_{1}, a_{2}, \cdots, a_{n}\right)$ is an $n$-tuple with elements of $a_{1}, a_{2}, \cdots, a_{n} .|\mathbb{A}|$ represents the cardinality of the set $\mathbb{A} \cdot \operatorname{diag}(\mathbf{a})$ is a diagonal matrix whose diagonal entries are equal to the elements of $\mathbf{a}$. The $M \times M$ identity matrix is denoted by $\mathbf{I}_{M} \cdot \operatorname{sgn}(x)$ denotes the sign function with $\operatorname{sgn}(x)=1$ for $x \geq 0$ and $\operatorname{sgn}(x)=-1$ otherwise. The real and image part of $a$ are denoted by $\Re\{a\}$ and $\Im\{a\}$, respectively. $\mathbb{E}\{$.$\} stands for the statistical expectation. \otimes$ and $\odot$ represent Kronecker and Khatri-Rao products, respectively. $\operatorname{vec}(\mathbf{A})=\left[\begin{array}{llll}\mathbf{a}_{1}^{T} & \mathbf{a}_{2}^{T} & \cdots & \mathbf{a}_{n}^{T}\end{array}\right]^{T}$ denotes the vectorization operation. $\mathbf{A}^{\dagger}$ and $\Pi_{\mathbf{A}}^{\perp}$ indicate the pseudoinverse and the projection matrix onto the null space of the full column rank matrix $\mathbf{A}^{H}$, respectively.

\section{SYSTEM MODEL}

We consider a SLA with $M$ elements located at positions $\left(m_{1} \frac{\lambda}{2}\right.$, $\left.m_{2} \frac{\lambda}{2}, \cdots, m_{M} \frac{\lambda}{2}\right)$ with $m_{i} \in \mathbb{M}$. Here, $\mathbb{M}$ is a set of integers with cardinality $|\mathrm{M}|=M$, and $\lambda$ denotes the wavelength of the incoming signals. $K$ narrowband signals with distinct DoAs $\boldsymbol{\theta}=$ $\left[\theta_{1}, \theta_{2}, \cdots, \theta_{K}\right]^{T}$ are assumed to impinge on the SLA from far field. While the estimation of the number of sources is an important problem, we assume perfect knowledge of the number of sources here. The unquantized array measurements at time instance $t$ can be modeled as

$$
\mathbf{y}(t)=\mathbf{A}(\boldsymbol{\theta}) \mathbf{s}(t)+\mathbf{n}(t) \in \mathbb{C}^{M \times 1}, \quad t=1, \cdots, N,
$$

where $\mathbf{s}(t) \in \mathbb{C}^{K \times 1}$ denotes the vector of source signals, $\mathbf{n}(t) \in$ $\mathbb{C}^{M \times 1}$ is additive noise, and $\mathbf{A}(\boldsymbol{\theta})=\left[\mathbf{a}\left(\theta_{1}\right), \mathbf{a}\left(\theta_{2}\right), \cdots \mathbf{a}\left(\theta_{K}\right)\right] \in$ $\mathbb{C}^{M \times K}$ represents the SLA steering matrix with

$$
\mathbf{a}\left(\theta_{i}\right)=\left[\begin{array}{llll}
e^{\mathrm{j} \pi \sin \theta_{i} m_{1}} & e^{\mathrm{j} \pi \sin \theta_{i} m_{2}} & \cdots & e^{\mathrm{j} \pi \sin \theta_{i} m_{M}}
\end{array}\right]^{T},
$$

being the SLA manifold vector for the $i^{\text {th }}$ signal. Further, the following assumptions are made on source signals and noise:

A1 $\mathbf{n}(t)$ follows a zero-mean circular complex Gaussian distribution with the covariance matrix $\mathbb{E}\left\{\mathbf{n}(t) \mathbf{n}^{H}(t)\right\}=\sigma^{2} \mathbf{I}_{M}$

A2 The source signal vector is modeled as a zero-mean circular complex Gaussian random vector with covariance matrix $\mathbb{E}\left\{\mathbf{s}(t) \mathbf{s}^{H}(t)\right\}=\operatorname{diag}(\mathbf{p})$ where $\mathbf{p}=\left[p_{1}, p_{2}, \cdots p_{K}\right]^{T} \in$ $\mathbb{R}_{>0}^{K \times 1}$ (i. e., $p_{l}>0, \forall l$ ).

A3 Source and noise vectors are mutually independent.

A4 There is no temporal correlation between the snapshots, i.e., $\mathbb{E}\left\{\mathbf{n}\left(t_{1}\right) \mathbf{n}^{H}\left(t_{2}\right)\right\}=\mathbb{E}\left\{\mathbf{s}\left(t_{1}\right) \mathbf{s}^{H}\left(t_{2}\right)\right\}=\mathbf{0}$ when $t_{1} \neq t_{2}$ and $\mathbf{0}$ is an all zero matrix of appropriate dimensions.

Based on the above assumptions, the covariance matrix of $\mathbf{y}(t)$ is expressed as

$\mathbf{R}=\mathbb{E}\left\{\mathbf{y}(t) \mathbf{y}^{H}(t)\right\}=\mathbf{A}(\boldsymbol{\theta}) \operatorname{diag}(\mathbf{p}) \mathbf{A}^{H}(\boldsymbol{\theta})+\sigma^{2} \mathbf{I}_{M} \in \mathbb{C}^{M \times M}$. (3) (a)

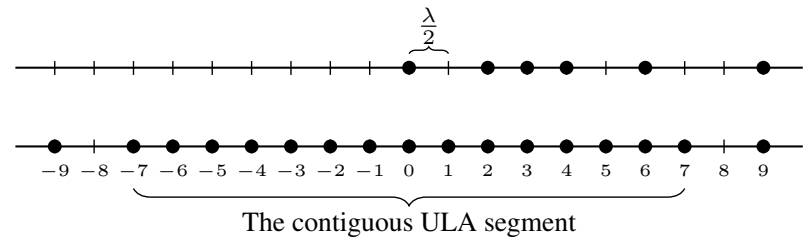

Fig. 1. Array geometry of a co-prime array with $M=6$ elements: (a) physical array with $\mathrm{M}=\{0,2,3,4,6,9\}$; (b) difference coarray with $\mathbb{D}=\{0,1,2,3,4,5,6,7,9\}$.

Vectorizing the covariance matrix in (3) leads to $[10,14,16]$

$$
\begin{aligned}
\mathbf{r} & \doteq \operatorname{vec}(\mathbf{R})=\left(\mathbf{A}^{*}(\boldsymbol{\theta}) \odot \mathbf{A}(\boldsymbol{\theta})\right) \mathbf{p}+\sigma^{2} \operatorname{vec}\left(\mathbf{I}_{M}\right), \\
& =\mathbf{J A}_{d}(\boldsymbol{\theta}) \mathbf{p}+\sigma^{2} \mathbf{J g} \in \mathbb{C}^{M^{2} \times 1},
\end{aligned}
$$

where $\mathbf{A}_{d}(\boldsymbol{\theta}) \in \mathbb{C}^{(2 D-1) \times K}$ corresponds to the steering matrix of the difference co-array of the SLA whose elements are located at $\left(-\ell_{D-1} \frac{\lambda}{2}, \cdots, 0, \cdots, \ell_{D-1} \frac{\lambda}{2}\right)$ with $\ell_{i} \in \mathbb{D}=\left\{\left|m_{p}-m_{q}\right|:\right.$ $\left.m_{p}, m_{q} \in \mathbb{M}\right\}$ and $D=|\mathbb{D}|$. Further, $\mathbf{g} \in\{0,1\}^{(2 D-1) \times 1}$ is a column vector with $[\mathbf{g}]_{i}=\delta[i-D]$, and the selection matrix $\mathbf{J} \in\{0,1\}^{M^{2} \times(2 D-1)}$ is represented as follows [10]

$$
\mathbf{J}=\left[\begin{array}{lllll}
\operatorname{vec}\left(\mathbf{L}_{D-1}^{T}\right) & \cdots & \operatorname{vec}\left(\mathbf{L}_{0}\right) & \cdots & \operatorname{vec}\left(\mathbf{L}_{D-1}\right),
\end{array}\right],
$$

where $\left[\mathbf{L}_{n}\right]_{p, q}=\left\{\begin{array}{lc}1, & \text { if } m_{p}-m_{q}=\ell_{n}, \\ 0, & \text { otherwise, }\end{array}\right.$ with $1 \leq p, q \leq M$ and $0 \leq n \leq D-1$.

The difference co-array model in (4) can be perceived to be the response of a virtual array whose steering matrix is given by $\mathbf{A}_{d}(\boldsymbol{\theta})$ to the parameter vector with signal powers $\mathbf{p}$ in presence of the noise vector $\sigma^{2} \operatorname{vec}\left(\mathbf{I}_{M}\right)$. This virtual array includes a contiguous ULA segment around the origin with the size of $2 v-1$ where $v$ is the largest integer such that $\{0,1, \cdots, v-1\} \subseteq \mathbb{D}$. An illustrative example of an SLA, the corresponding difference co-array and its contiguous ULA segment is provided in Fig. 1. It has been shown in [8-10] that by properly designing SLAs and exploiting the resulting structure of the source signal covariance matrix efficiently, SLAs are capable of identifying more sources than the number of physical elements in the array.

Here it is assumed that each array element is connected to a onebit ADC which directly converts the received analog signal into binary data by comparing the real and imaginary parts of the received signal individually with zero. In such a case, the one-bit measurements at the $m^{\text {th }}$ array element is given by

$$
\begin{aligned}
{[\mathbf{x}(t)]_{m}=} & Q\left([\mathbf{y}(t)]_{m}\right)=\frac{1}{\sqrt{2}} \operatorname{sgn}\left(\Re\left([\mathbf{y}(t)]_{m}\right)\right) \\
& +\mathrm{j} \frac{1}{\sqrt{2}} \operatorname{sgn}\left(\Im\left([\mathbf{y}(t)]_{m}\right)\right) .
\end{aligned}
$$

The problem under consideration is estimation of source DoAs using one-bit quantized measurements, i.e., $\mathbf{X}=[\mathbf{x}(1), \mathbf{x}(t), \cdots, \mathbf{x}(N-$ 1)], collected by the SLA.

\section{ONE-BIT CO-ARRAY-BASED MUSIC}

In this section, we review the one-bit co-array-based MUSIC algorithm proposed in [26]. This algorithm is based on the arcsin law [28] which establishes a relationship between the covariance matrix of one-bit data and the covariance matrix of unquantized data. 
Indeed, it follows from the arcsin law that [26],

$$
\mathbf{R}_{\mathbf{x}}=\mathbb{E}\left\{\mathbf{x}(t) \mathbf{x}^{H}(t)\right\}=\frac{2}{\pi} \operatorname{arcsine}(\overline{\mathbf{R}}),
$$

where $\overline{\mathbf{R}}=\frac{1}{\sigma^{2}+\sum_{k=1}^{K} p_{k}} \mathbf{R}$ denotes the normalized covariance matrix of $\mathbf{y}(t)$ and $[\operatorname{arcsine}(\overline{\mathbf{R}})]_{m, n}=\arcsin \left(\Re\left\{[\mathbf{R}]_{m, n}\right\}\right)+$ $\mathrm{j} \arcsin \left(\Im\left\{[\overline{\mathbf{R}}]_{m, n}\right\}\right)$. Reformulating (7) gives the normalized covariance matrix of $\mathbf{y}(t)$ based on the covariance matrix of one-bit data as follows:

$$
\overline{\mathbf{R}}=\operatorname{sine}\left(\frac{\pi}{2} \mathbf{R}_{\mathbf{x}}\right)
$$

where $\left[\operatorname{sine}\left(\frac{\pi}{2} \mathbf{R}_{\mathbf{x}}\right)\right]_{m, n}=\sin \left(\frac{\pi}{2} \Re\left\{[\overline{\mathbf{R}}]_{m, n}\right\}\right)+\mathrm{j} \sin \left(\frac{\pi}{2} \Im\left\{[\overline{\mathbf{R}}]_{m, n}\right\}\right)$ Accordingly, an estimate of the normalized covariance matrix of $\mathbf{y}(t)$ can be derived as [26]

$$
\widehat{\overline{\mathbf{R}}}=\operatorname{sine}\left(\frac{\pi}{2} \widehat{\mathbf{R}}_{\mathbf{x}}\right) .
$$

where $\widehat{\mathbf{R}}_{\mathbf{x}}=\frac{1}{N} \mathbf{X X} \mathbf{X}^{H}$ is the sample covariance matrix of the onebit data. Then, the normalized ASCM can be constructed from $\widehat{\mathbf{R}}$ as [26]

$$
\widehat{\mathbf{R}}_{v}=\left[\begin{array}{llll}
\mathbf{T}_{v} \mathbf{J}^{\dagger} \widehat{\overline{\mathbf{r}}} & \mathbf{T}_{v-1} \mathbf{J}^{\dagger} \hat{\overline{\mathbf{r}}} & \ldots & \mathbf{T}_{1} \mathbf{J}^{\dagger} \overline{\overline{\mathbf{r}}}
\end{array}\right] \in \mathbb{C}^{v \times v},
$$

where $\widehat{\overline{\mathbf{r}}}=\operatorname{vec}(\widehat{\overline{\mathbf{R}}})$ and $\mathbf{T}_{i}$ is a selection matrix, defined as

$$
\mathbf{T}_{i}=\left[\begin{array}{lll}
\mathbf{0}_{v \times(i+D-v-1)} & \mathbf{I}_{v} & \mathbf{0}_{v \times(D-i)}
\end{array}\right] \in\{0,1\}^{v \times(2 D-1)} .
$$

Finally, the DoA estimates are obtained by applying MUSIC to $\widehat{\mathbf{R}}_{v}$.

\section{ASYMPTOTIC PERFORMANCE ANALYSIS}

In this section, we investigate the asymptotic performance of OBCAB-MUSIC through the derivation of a closed form expression for the covariance matrix of the DoA estimation errors. Our main results are summarized in Theorem 1, Corollary 1 and Theorem 2.

Theorem 1. The asymptotic expression (as $N \rightarrow \infty$ ) for the covariance between the DoA estimation errors obtained by $O B C A B$ MUSIC is given by

$$
\mathcal{E}_{\theta_{k_{1}}, \theta_{k_{2}}}=\frac{\left(\sigma^{2}+\sum_{k=1}^{K} p_{k}\right)^{2} \Re\left\{\mathbf{z}_{k_{1}}^{H} \mathbf{T} \mathbf{J}^{\dagger} \mathbf{M} \mathbf{J}^{\dagger H} \mathbf{T}^{H} \mathbf{z}_{k_{2}}\right\}}{N \pi^{2} p_{k_{1}} p_{k_{2}} q_{k_{1}} q_{k_{2}} \cos \theta_{k_{1}} \cos \theta_{k_{2}}},
$$

where

$$
\begin{aligned}
& \mathbf{T}=\left[\begin{array}{llll}
\mathbf{T}_{v}^{T} & \mathbf{T}_{v-1}^{T} & \cdots & \mathbf{T}_{1}^{T}
\end{array}\right]^{T} \in \mathbb{C}^{v^{2} \times(2 D-1)}, \\
& \mathbf{z}_{k}=\boldsymbol{\beta}_{k} \otimes \boldsymbol{\alpha}_{k}, \\
& \boldsymbol{\beta}_{k}=\Pi_{\mathbf{A}_{v}(\boldsymbol{\theta})}^{\perp} \operatorname{diag}(\mathbf{v}) \mathbf{a}\left(\theta_{k}\right), \\
& \boldsymbol{\alpha}_{k}=\mathbf{A}_{v}^{\dagger T}(\boldsymbol{\theta}) \mathbf{e}_{k}, \\
& q_{k}=\mathbf{a}^{H}\left(\theta_{k}\right) \operatorname{diag}(\mathbf{v}) \Pi_{\mathbf{A}_{v}}^{\perp} \operatorname{diag}(\mathbf{v}) \mathbf{a}\left(\theta_{k}\right), \\
& {[\mathbf{M}]_{p, q}=\frac{1}{2}\left(\sqrt{1-\left[\Re\left\{[\overline{\mathbf{r}}]_{p}\right\}\right]^{2}} \times \sqrt{1-\left[\Re\left\{[\overline{\mathbf{r}}]_{q}\right\}\right]^{2}}\right.} \\
& \left.+\sqrt{1-\left[\Im\left\{[\overline{\mathbf{r}}]_{p}\right\}\right]^{2}} \times \sqrt{1-\left[\Im\left\{[\overline{\mathbf{r}}]_{q}\right\}\right]^{2}}\right) \Re\left\{[\boldsymbol{\Sigma}]_{p, q}\right\} \\
& +\frac{\mathrm{j}}{2}\left(\sqrt{1-\left[\Im\left\{[\overline{\mathbf{r}}]_{p}\right\}\right]^{2}} \times \sqrt{1-\left[\Re\left\{[\overline{\mathbf{r}}]_{q}\right\}\right]^{2}}\right. \\
& \left.+\sqrt{1-\left[\Re\left\{[\overline{\mathbf{r}}]_{p}\right\}\right]^{2}} \times \sqrt{1-\left[\Im\left\{[\overline{\mathbf{r}}]_{q}\right\}\right]^{2}}\right) \Im\left\{[\boldsymbol{\Sigma}]_{p, q}\right\},
\end{aligned}
$$

with $\overline{\mathbf{r}}=\operatorname{vec}(\overline{\mathbf{R}})$ and $\boldsymbol{\Sigma}=\mathbb{E}\left\{\left[\operatorname{vec}\left(\widehat{\mathbf{R}}_{\mathbf{x}}\right)-\operatorname{vec}\left(\mathbf{R}_{\mathbf{x}}\right)\right]\left[\operatorname{vec}\left(\widehat{\mathbf{R}}_{\mathbf{x}}\right)-\right.\right.$ $\left.\left.\operatorname{vec}\left(\mathbf{R}_{\mathbf{x}}\right)\right]^{H}\right\} \in \mathbb{C}^{M^{2} \times M^{2}}$.

We note that we have derived a closed-form expression for $\boldsymbol{\Sigma}$ by computing the fourth order moments of the orthant probability. Unfortunately, this closed-form expression is rather cumbersome and is derived for each element; we do not provide this expression of $\boldsymbol{\Sigma}$ due to the lack of space but we refer the interested readers to the journal extension of this work [29]. This closed form-expression shows that $\boldsymbol{\Sigma}$ is only a function of $\overline{\mathbf{r}}$.

Corollary 1. The asymptotic expression (as $N \rightarrow \infty$ ) for the Mean Square Error (MSE) of the DoA estimates obtained by OBCABMUSIC is given by

$$
\mathcal{E}_{\theta_{k}}=\frac{\left(\sigma^{2}+\sum_{k^{\prime}=1}^{K} p_{k^{\prime}}\right)^{2} \mathbf{z}_{k}^{H} \mathbf{T} \mathbf{J}^{\dagger} \mathbf{M} \mathbf{J}^{\dagger H} \mathbf{T}^{H} \mathbf{z}_{k}}{N \pi^{2} p_{k}^{2} q_{k}^{2} \cos ^{2} \theta_{k}} .
$$

Remark 1. It is concluded from Corollary 1 that, similar to Infinite-Bit Co-Array-Based MUSIC (IBCAB-MUSIC), the MSE of OBCAB-MUSIC also depends on both the physical and the virtual array geometries.

Remark 2. Another interesting implication of Corollary 1 is that the MSE of OBCAB-MUSIC reduces at the same rate as that of IBCABMUSIC with respect to $N$; i.e. $\mathcal{E}_{\theta_{k}} \propto \frac{1}{N}$ for both.

Remark 3. It is readily clear from the definition that $\overline{\mathbf{r}}$ is a function of the SNR not $\mathbf{p}$ and $\sigma^{2}$. This indicates that $\mathbf{M}$ is a function of the SNR instead of $\mathbf{p}$ and $\sigma^{2}$. Further, multiplying the numerator and denominator of $\left(\sigma^{2}+\sum_{k^{\prime}=1}^{K} p_{k^{\prime}}\right)^{2} / p_{k}^{2}$ by $1 / \sigma^{4}$ proves that it is also a function of the SNR not $\mathbf{p}$ and $\sigma^{2}$. This implies that the MSE of OBCAB-MUSIC is a function of the SNR instead of $\mathbf{p}$ and $\sigma^{2}$.

Theorem 2. Assume all sources have equal power $p$ and $S N R=$ $p / \sigma^{2}$. Then, for a sufficiently large SNR, the MSE converges to the following constant value:

$$
\lim _{S N R \rightarrow \infty} \mathcal{E}_{\theta_{k}}=\frac{K^{2} \mathbf{z}_{k}^{H} \mathbf{T} \mathbf{J}^{\dagger} \overline{\mathbf{M}} \mathbf{J}^{\dagger H} \mathbf{T}^{H} \mathbf{z}_{k}}{N \pi^{2} \cos ^{2} \theta_{k} q_{k}^{2}}>0,
$$

where $\overline{\mathbf{M}}$ is obtained by replacing $\overline{\mathbf{r}}$ in the definitions of $\mathbf{M}$ and $\mathbf{\Sigma}$ (kindly refer to Theorem 1) with $\widetilde{\mathbf{r}}$ which is defined as

$$
\widetilde{\mathbf{r}}=\mathbf{J}\left[\frac{1}{K} \mathbf{A}_{d}(\boldsymbol{\theta}) \mathbf{1}_{M}+\left(1-\frac{1}{K}\right) \mathbf{g}\right] \in \mathbb{C}^{M^{2} \times 1},
$$

where $\mathbf{1}_{M}$ is the $M \times 1$ all-one vector.

Remark 4. It follows from Theorem 2 that it is not possible to make the MSE of OBCAB-MUSIC arbitrarily small by increasing the SNR.

\section{SIMULATION RESULTS}

In this section, we provide some numerical results to validate the asymptotic closed-form expression derived for the performance of OBCAB-MUSIC, as well as to show a representative use case of predicting probability of resolution. In all simulation results, each simulated point has been computed by 1000 Monte Carlo repetitions. In addition, it is assumed that the $K$ independent sources are located at $\left\{-60^{\circ}+120^{\circ}(k-1) /(K-1): k=0,1, \cdots, K-1\right\}$. All sources have an equal power, i.e., $p_{k}=p$ for all $k$, and the SNR is defined as $10 \log \frac{p}{\sigma^{2}}$. Throughout this section, we use a nested array with $M=10$ physical elements and the following geometry:

$$
\mathbb{M}_{\text {nested }}:\{1,2,3,4,5,8,10,15,20\} \text {. }
$$




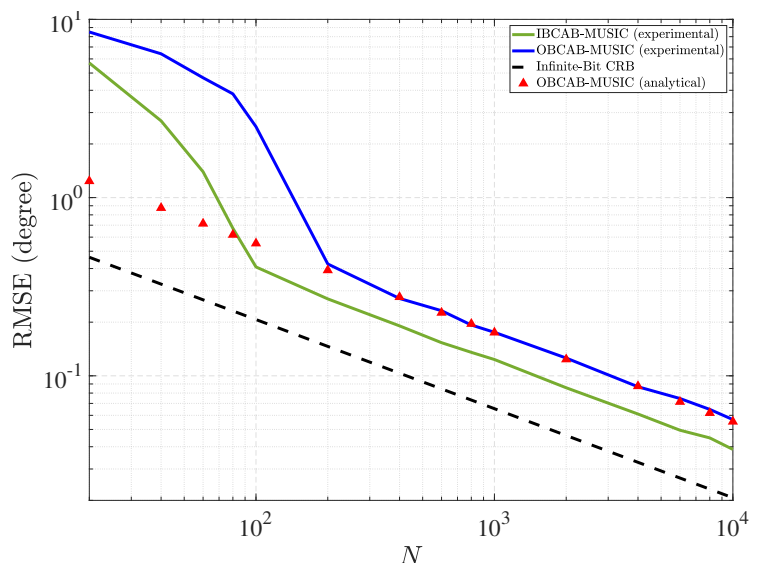

Fig. 2. RMSE in degree for $\theta_{2}$ versus the number of snapshots for a nested array with $M=10$ elements and configuration given in (22), $\mathrm{SNR}=3 \mathrm{~dB}$, and $K=12>M$.

Fig. 2 depicts the Root-Mean-Squares-Error (RMSE) for $\theta_{2}$ in degree versus the number of snapshots for the nested array in (22). The SNR is assumed to be $3 \mathrm{~dB}$ and the number of sources is considered to be $K=12>M$. Interestingly, Fig. 2 illustrates a close agreement between the numerical results and the asymptotic analytical RMSE expression for OBCAB-MUSIC when about 200 or more snapshots are available. Fig. 2 also shows that when a small number of snapshots is available, for example less than 200, both IBCAB-MUSIC and OBCAB-MUSIC are confronted with substantial performance degradation. This performance loss is justified by the subspace swap arising from the inaccurate estimate of the resulting augmented covariance matrix. In addition, it is observed that the RMSEs of IBCAB-MUSIC and OBCAB-MUSIC reduce at an identical rate by increasing $N$. This observation is consistent with Remark 2.

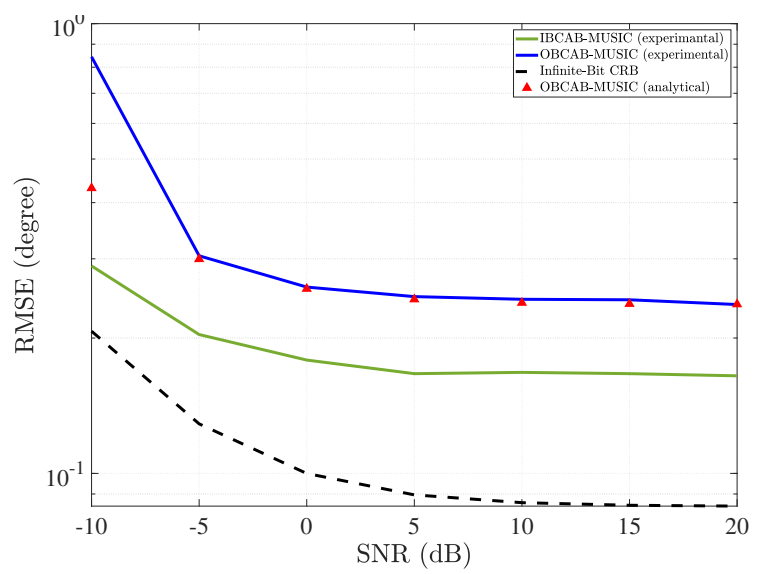

Fig. 3. RMSE in degree for $\theta_{2}$ versus the SNR for a nested array with $M=10$ elements and configuration given in (22), $N=500$, and $K=12>M$.

Fig. 3 shows the RMSE for $\theta_{2}$ in degree versus the SNR for the same setup used for Fig. 2. The number of snapshots is considered to be $N=500$. It is observed that there is a close agreement between the analytical RMSE and simulation results. Further, we see that the RMSE of OBCAB-MUSIC converges to a constant value as SNR $\rightarrow$ $\infty$ which is consistent with Theorem 2 and Remark 4 .

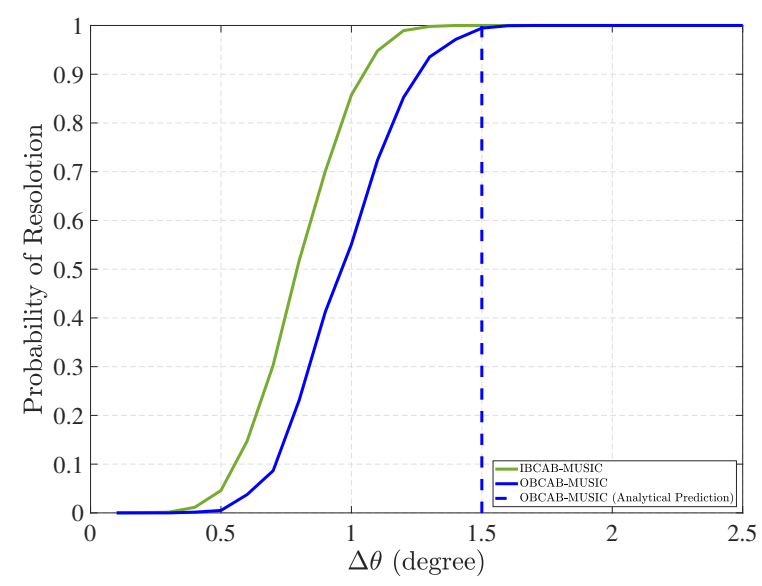

Fig. 4. Probability of resolution versus source separation in degree for a nested array with $M=10$ elements and configuration given in (22), $N=500$ and $\mathrm{SNR}=0 \mathrm{~dB}$.

Fig. 4 depicts the probability of resolution versus the source separation for IBCAB-MUSIC and OBCAB-MUSIC when the nested array given in (22) is employed. The number of snapshots and the SNR are considered to be $N=500$ and $0 \mathrm{~dB}$, respectively. In addition, we consider two sources with equal powers, located at $\theta_{1}=20^{\circ}-\frac{\Delta \theta}{2}$ and $\theta_{2}=20^{\circ}+\frac{\Delta \theta}{2}$. We define the two sources as being resolvable if $\max _{i \in\{1,2\}}\left|\hat{\theta}_{i}-\theta_{i}\right|<\frac{\Delta \theta}{2}$ [30]. According to this definition and making use of two-dimensional Chebychev's bound [31], the probability of resolution can be lower bounded as

$$
\begin{aligned}
\mathbb{P}\left(\max _{i \in\{1,2\}}\left|\hat{\theta}_{i}-\theta_{i}\right|<\frac{\Delta \theta}{2}\right) \\
=\mathbb{P}\left(\left|\hat{\theta}_{1}-\theta_{1}\right|<\frac{\Delta \theta}{2},\left|\hat{\theta}_{2}-\theta_{2}\right|<\frac{\Delta \theta}{2}\right) \geq 1-\frac{2\left[\mathcal{E}\left(\theta_{1}\right)+\mathcal{E}\left(\theta_{2}\right)\right]}{\Delta \theta^{2}} \\
+\frac{2 \sqrt{\mathcal{E}_{\theta_{1}}^{2}+\mathcal{E}_{\theta_{2}}^{2}+2 \mathcal{E}_{\theta_{1}} \mathcal{E}_{\theta_{2}}-4 \mathcal{E}_{\theta_{1}, \theta_{2}}^{2}}}{\Delta \theta^{2}}
\end{aligned}
$$

where $\mathcal{E}\left(\theta_{1}\right), \mathcal{E}\left(\theta_{2}\right)$ and $\mathcal{E}\left(\theta_{1}, \theta_{2}\right)$ are given in (19) and (12). The analytical expression on the right hand side of (23) enables us to predict the minimum source separation required for achieving a particular probability of resolution. For example, Fig. 4 shows the predicted value for the the minimum source separation to achieve a probability of resolution greater than 0.9 , obtained from (23). It is observed that the predicted minimum source separation, which is $\Delta \theta=1.5^{\circ}$, is in a good agreement with the value obtained from the numerical simulations, which is $\Delta \theta=1.3^{\circ}$.

\section{CONCLUSION}

In this paper, we derived an asymptotic closed-form expression for the performance of One-Bit Co-Array-Based MUSIC (OBCABMUSIC). This analytical performance enables us to provide some insights inot the performance of OBCAB-MUSIC. For example, it was shown that the MSE of OBCAB-MUSIC reduces at the same rate as that of IBCAB-MUSIC with respect to the number of samples and, moreover, that the MSE of OBCAB-MUSIC converges to a constant value by increasing the SNR. Further, numerical simulations were provided to validate the asymptotic closed-form expression for the performance of OBCAB-MUSIC and to show an interesting use case of it in evaluating the resolution of OBCAB-MUSIC. 


\section{REFERENCES}

[1] H. Van Trees, Optimum Array Processing (Detection, Estimation, and Modulation Theory, Part IV). New York: John Wiley and Sons Inc., 2002.

[2] S. S. Haykin, J. Litva, , and T. J. Shepherd, Eds., Radar Array Processing. Berlin, Germany: Springer-Verlag, 1993.

[3] B. Ottersten, "Array processing for wireless communications," in Proceedings of 8th Workshop on Statistical Signal and Array Processing, Jun 1996, pp. 466-473.

[4] A. Paulraj, B. Ottersten, R. Roy, A. Swindlehurst, G. Xu, and T. Kailath, "16 subspace methods for directions-of-arrival estimation," Handbook of Statistics, vol. 10, pp. 693-739, 1993.

[5] F. Li, H. Liu, and R. J. Vaccaro, "Performance analysis for DoA estimation algorithms: unification, simplification, and observations," IEEE Trans. Aerosp. Electron. Syst, vol. 29, no. 4, pp. 1170-1184, Oct 1993.

[6] P. Stoica and A. Nehorai, "Performance study of conditional and unconditional direction-of-arrival estimation," IEEE Transactions on Acoustics, Speech, and Signal Processing, vol. 38, no. 10, pp. 1783-1795, Oct 1990.

[7] A. Moffet, "Minimum-redundancy linear arrays," IEEE Transactions on Antennas and Propagation, vol. 16, no. 2, pp. 172175, Mar 1968.

[8] P. P. Vaidyanathan and P. Pal, "Sparse sensing with co-prime samplers and arrays," IEEE Trans. Signal Process., vol. 59, no. 2, pp. 573-586, Feb 2011.

[9] P. Pal and P. P. Vaidyanathan, "Nested arrays: A novel approach to array processing with enhanced degrees of freedom," IEEE Transactions on Signal Processing, vol. 58, no. 8, pp. 4167-4181, Aug 2010.

[10] C. L. Liu and P. P. Vaidyanathan, "Cramér-Rao bounds for coprime and other sparse arrays, which find more sources than sensors," Digital Signal Processing, vol. 61, pp. 43 - 61, 2017.

[11] Y. D. Zhang, M. G. Amin, and B. Himed, "Sparsity-based DoA estimation using co-prime arrays," in 2013 IEEE International Conference on Acoustics, Speech and Signal Processing, May 2013, pp. 3967-3971.

[12] Z. Tan and A. Nehorai, "Sparse direction of arrival estimation using co-prime arrays with off-grid targets," IEEE Signal Processing Letters, vol. 21, no. 1, pp. 26-29, Jan 2014.

[13] Z. Yang, L. Xie, and C. Zhang, "A discretization-free sparse and parametric approach for linear array signal processing," IEEE Transactions on Signal Processing, vol. 62, no. 19, pp. 4959-4973, Oct 2014.

[14] M. Wang and A. Nehorai, "Coarrays, MUSIC, and the CramérRao bound," IEEE Trans. Signal Process., vol. 65, no. 4, pp. 933-946, Feb 2017.

[15] S. Sedighi, M. R. Bhavani Shankar, and B. Ottersten, "A statistically efficient estimator for co-array based doa estimation," in 2018 52nd Asilomar Conference on Signals, Systems, and Computers, 2018, pp. 880-883.

[16] S. Sedighi, B. S. M. R. Rao, and B. Ottersten, "An asymptotically efficient weighted least squares estimator for co-arraybased DoA estimation," IEEE Transactions on Signal Processing, vol. 68, pp. 589-604, 2020.
[17] L. Lu, G. Y. Li, A. L. Swindlehurst, A. Ashikhmin, and R. Zhang, "An overview of massive MIMO: Benefits and challenges," IEEE Journal of Selected Topics in Signal Processing, vol. 8, no. 5, pp. 742-758, Oct 2014.

[18] S. Jacobsson, G. Durisi, M. Coldrey, U. Gustavsson, and C. Studer, "One-bit massive MIMO: Channel estimation and high-order modulations," in 2015 IEEE International Conference on Communication Workshop (ICCW), June 2015, pp. 1304-1309.

[19] S. Rao, A. Mezghani, and A. L. Swindlehurst, "Channel estimation in one-bit massive MIMO systems: Angular versus unstructured models," IEEE Journal of Selected Topics in Signal Processing, vol. 13, no. 5, pp. 1017-1031, Sep. 2019.

[20] A. Ameri, A. Bose, J. Li, and M. Soltanalian, "One-bit radar processing with time-varying sampling thresholds," IEEE Transactions on Signal Processing, vol. 67, no. 20, pp. 52975308, Oct 2019.

[21] J. Li, M. M. Naghsh, S. J. Zahabi, and M. Modarres-Hashemi, "Compressive radar sensing via one-bit sampling with timevarying thresholds," in 2016 50th Asilomar Conference on Signals, Systems and Computers, Nov 2016, pp. 1164-1168.

[22] O. Bar-Shalom and A. J. Weiss, "DoA estimation using onebit quantized measurements," IEEE Transactions on Aerospace and Electronic Systems, vol. 38, no. 3, pp. 868-884, July 2002.

[23] C. Stöckle, J. Munir, A. Mezghani, and J. A. Nossek, "1-bit direction of arrival estimation based on compressed sensing," in 2015 IEEE 16th International Workshop on Signal Processing Advances in Wireless Communications (SPAWC), June 2015, pp. 246-250.

[24] M. Stein, K. Barbe, and J. A. Nossek, "DoA parameter estimation with 1-bit quantization bounds, methods and the exponential replacement," in WSA 2016; 20th International ITG Workshop on Smart Antennas, March 2016, pp. 1-6.

[25] X. Huang and B. Liao, "One-bit MUSIC," IEEE Signal Processing Letters, vol. 26, no. 7, pp. 961-965, July 2019.

[26] C. Liu and P. P. Vaidyanathan, "One-bit sparse array DoA estimation," in 2017 IEEE International Conference on Acoustics, Speech and Signal Processing (ICASSP), March 2017, pp. 3126-3130.

[27] S. Sedighi, B. Shankar, M. Soltanalian, and B. Ottersten, "Onebit doa estimation via sparse linear arrays," in ICASSP 2020 2020 IEEE International Conference on Acoustics, Speech and Signal Processing (ICASSP), 2020, pp. 9135-9139.

[28] J. H. Van Vleck and D. Middleton, "The spectrum of clipped noise," Proceedings of the IEEE, vol. 54, no. 1, pp. 2-19, 1966.

[29] S. Sedighi, M. Shankar, M. Soltanalian, and B. Ottersten, "On the performance of one-bit doa estimation via sparse linear arrays," arXiv preprint arXiv:2012.14051, 2020.

[30] M. Kaveh and A. Barabell, "The statistical performance of the MUSIC and the minimum-norm algorithms in resolving plane waves in noise," IEEE Transactions on Acoustics, Speech, and Signal Processing, vol. 34, no. 2, pp. 331-341, April 1986.

[31] D. N. Lal, "A note on a form of Tchebycheff's inequality for two or more variables," Sankhyā: The Indian Journal of Statistics (1933-1960), vol. 15, no. 3, pp. 317-320, 1955. 